\title{
Composición y variabilidad del ictioplancton de la laguna El Quelele, Nayarit, México
}

\author{
Ichthyoplankton composition and variability of the laguna \\ El Quelele, Nayarit, Mexico
María del Carmen Navarro-Rodríguez ${ }^{1}$, Luis Fernando González Guevara1, Ramiro Flores- Vargas $^{2}$, María Elena González Ruelas ${ }^{1}$ y Fátima Maciel Carrillo González ${ }^{1}$ \\ ${ }^{1}$ Centro Universitario de la Costa, Campus Vallarta, Departamento de Ciencias, Universidad de Guadalajara, Av. Universidad, No. \\ 203 Delegación Ixtapa Puerto Vallarta C.P. 48280, Jalisco, México. (UDG-CA-345) \\ ${ }^{2}$ Centro Universitario de la Costa Sur, Departamento de Estudios para el Desarrollo Sustentable de Zonas Costeras, Universidad de \\ Guadalajara, Gómez Farías No. 82 San Patricio-Melaque, Jalisco, México. (UDG-CA-341) \\ carmenna@universo.com
}

\begin{abstract}
Resumen.- Se analizó la variación espacio temporal de la abundancia del ictioplancton en la laguna El Quelele, bahía de Banderas, Nayarit. Se realizaron 20 arrastres zooplanctónicos diurnos, estacionalmente de primavera a invierno del 2001. De un total de 20 muestras de zooplancton se obtuvieron 224,4 larvas de peces las cuales estuvieron representadas por seis familias, ocho géneros y seis especies, siendo Engraulis mordax la especie más abundante (68 org. $1000 \mathrm{~m}^{-3}$ ), seguida de Dormitator latifroms (37 org. $1000 \mathrm{~m}^{-3}$ ) y Trachinotus rhodopus (18 org. $1000 \mathrm{~m}^{-3}$ ). Las variaciones de la abundancia espacio temporal estuvieron influenciadas por la temperatura $\left(29,8-31,6^{\circ} \mathrm{C}\right)$ y salinidad superficiales (11-34,8 psu) así como por las condiciones de marea y época del año. Las mayores concentraciones se presentaron durante otoño (81 org. 1000 $\mathrm{m}^{-3}$ ) e invierno (43 org. $1000 \mathrm{~m}^{-3}$ ), asociadas a la época fría y las menores abundancias en primavera (21 org. $1000 \mathrm{~m}^{-3}$ ) y verano asociadas a la época cálida con abundantes lluvias; en verano no se obtuvieron larvas. El índice de Shannon indicó que el sitio de muestreo 1 presentó el valor más alto de diversidad $(1,47)$, en tanto que, por estación del año, la diversidad fue prácticamente homogénea. El análisis de cluster mostró una mayor afinidad entre los sitios de muestreo 2 y 3, con un valor de 1,05 y entre los meses fríos (otoño e invierno), con un valor de 0,83 .
\end{abstract}

Palabras clave: Larvas de peces, diversidad, variación espaciotemporal, parámetros ambientales, Pacífico noreste

\begin{abstract}
This study presents an analysis of a spatial and temporal variation of ichthyoplankton abundance in the lagoon El Quelele, Bahía de Banderas, Nayarit. Twenty diurnal plankton tows were conducted seasonally from spring to winter 2001. Of twenty total samples of zooplankton 224,4 fish larvae were obtained, represented by six families eight genera and six species, being Engraulis mordax the most abundant species (68 org. $\mathrm{m}^{-3}$ ), followed by Dormitator latifroms (37 org. $\mathrm{m}^{-3}$ ) and Trachinotus rhodopus (18 org. $\mathrm{m}^{-3}$ ). Variations of the spatio-temporal abundance were influenced by surface temperature $\left(29,8-31,6^{\circ} \mathrm{C}\right)$ and salinity (11-34,8 psu) as well as by tidal conditions and season. The highest concentrations occurred during autumn ( 81 org. $\mathrm{m}^{-3}$ ) and winter (43 org. $\mathrm{m}^{-3}$ ) associated to the cold season, and smallest abundances in spring (21 org. $\mathrm{m}^{-3}$ ) and summer associated to the warm season and abundant rains; in summer, no larvae were captured. The Shannon index showed that site of sampling 1 presented the highest diversity value $(1,47)$ whereas, by season diversity was practically homogenous. Cluster analysis showed greater affinity between sites 2 and 3 $(1,05)$ and between cold months (autumn and winter) with a value of 0,83 .
\end{abstract}

Key words: Fish larvae, diversity, spatial and temporal patterns, environmental factors, Pacific northeast

\section{Introducción}

México posee a lo largo de sus litorales aproximadamente 130 lagunas costeras que exhiben diferentes tamaños, regímenes hidrológicos, biota, hábitos y flujos de energía, siendo consideradas éstas, entre los ecosistemas más productivos de la biosfera, debido a su alta productividad pesquera (FloresVerdugo 1989, Contreras 1993, Suárez Morales 1994) y porque juegan además, un papel primordial en las estrategias de reproducción de los peces como áreas propicias para los adultos en reproducción y de disponibilidad de alimento para el desarrollo de los 
juveniles (Funes et al. 1998). El ictioplancton es, sin duda, el componente más importante dentro de las comunidades planctónicas ya que es el que presenta la mayor relevancia en términos de potencialidad pesquera; es por ello que, en el país, la evaluación de los estudios larvarios de peces son necesarios e importantes para la estimación del potencial comercial de los recursos pesqueros que existen en áreas poco conocidas, y por medio de esta alternativa, determinar los recursos pesqueros susceptibles de ser explotados y establecer las medidas para su aprovechamiento sustentable.

\section{Área de estudio}

La laguna El Quelele es un sistema costero que se encuentra en la bahía de Banderas, en la parte suroeste de la costa de Nayarit y al noroeste de Jalisco, entre los $105^{\circ} 17^{\prime} \mathrm{W}$ y $20^{\circ} 43^{\prime} \mathrm{N}$, a $15 \mathrm{~km}$ hacia el norte de Puerto Vallarta, Jalisco (Fig. 1). Tiene un área total de planos lodosos de 100 hectáreas que son inundadas periódicamente por efectos de las mareas, y por el agua de las precipitaciones pluviales y escurrimientos durante las temporadas de lluvias (junio-septiembre), presentando su nivel máximo de agua y alcanzando una profundidad promedio de 1,20 m. Este cuerpo de agua se conecta al océano a través del estero El Chino, cuya boca fue modificada al construirse la rada portuaria de Nuevo Vallarta, Nayarit (Cupul-Magaña 1999, 2000). La laguna está ubicada dentro de una zona climática semicálida subhúmeda donde la temperatura y la precipitación pluvial promedio anual oscilan entre 26 y $28^{\circ} \mathrm{C}$ y entre 930,8 a $1660 \mathrm{~mm}$, respectivamente (García 1973). El tipo de vegetación dominante es el manglar siendo la principal especie Laguncularia racemosa (L.) Gaertner S. (Cupul-Magaña 1999). El complejo lacustre El Quelele aun conserva la mayor parte de sus características naturales; se destaca por albergar una infinidad de seres vivos, presenta una vegetación silvestre relativamente conservada, destacando sobre los márgenes de la laguna principalmente el mangle blanco (L. racemosa), cubriendo un área de 33,54 ha y con un promedio de 4'537,63 árboles ha ${ }^{-1}$ (Gobierno del Estado de Jalisco 1999).

\section{Materiales y métodos}

En la zona de estudio fueron ubicados cinco sitios de muestreo abarcando una longitud total de 11,5 km (Fig. 1), con una distancia aproximada entre sitios de $2,3 \mathrm{~km}$, realizándose 20 arrastres, con periodicidad estacional, de primavera a invierno en 2001.

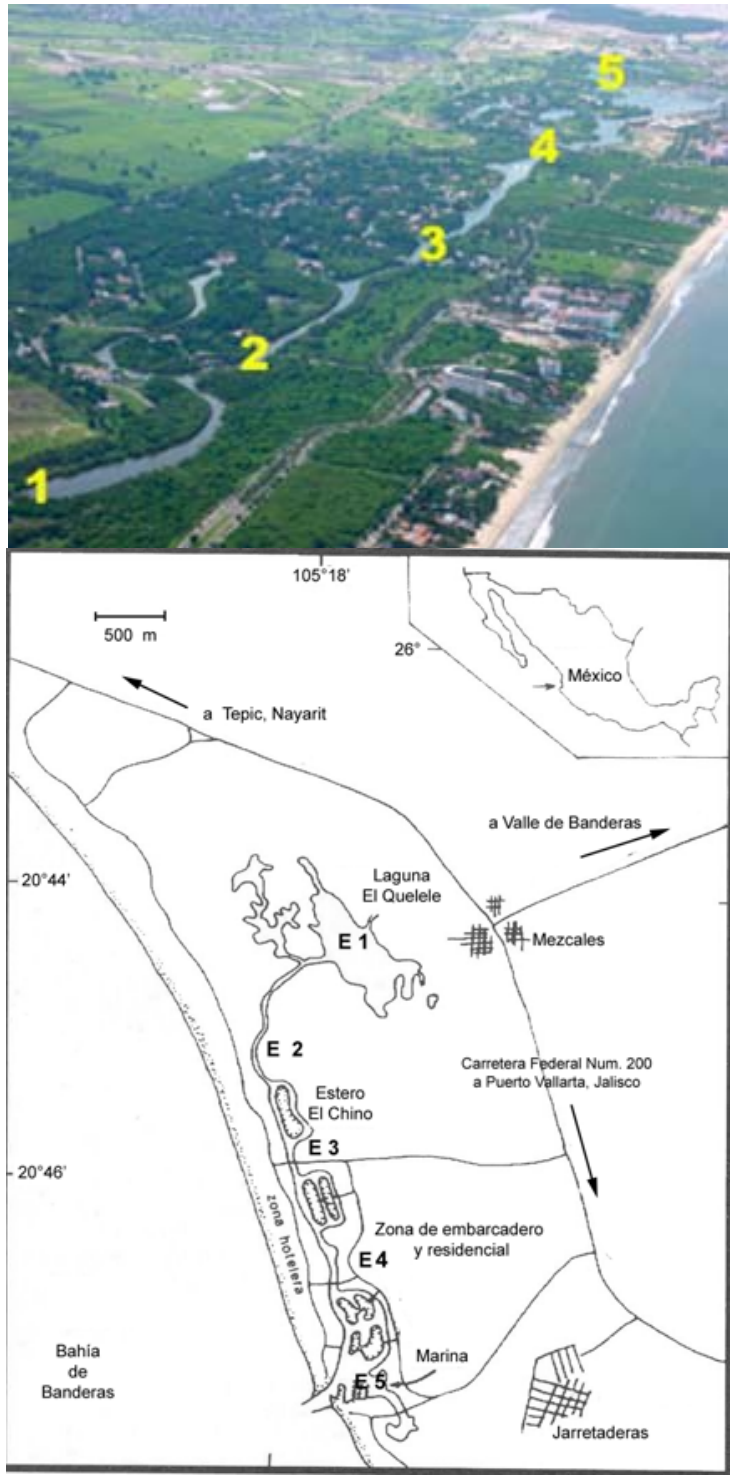

Figura 1

Área de estudio y sitios de muestreo (1, 2, 3, 4 y 5) en la laguna El Quelele, Nayarit, México (primavera-invierno 2001)

Study area and sampling sites (1, 2, 3, 4 and 5) in the laguna El Quelele, Nayarit, Mexico (spring-winter 2001)

Las muestras de zooplancton se obtuvieron mediante arrastres diurnos efectuados a bordo de una lancha con motor fuera de borda, por medio de una red tipo “Zeppelin”, con una manga de $505 \mu \mathrm{m}$ de luz de malla por 1,50 m de longitud y 0,60 m de diámetro de la boca, y equipada con un flujómetro digital (General Oceanics Inc. 2030R), para medir el flujo del agua filtrada. Los arrastres fueron superficiales, con una duración de 10 
minutos, y bajo condiciones de marea alta, apoyándose en las tablas de marea emitidas por el Departamento de Oceanografía Física del CICESE (Centro de Investigación Científica y Educación Superior de Ensenada). El material recolectado se colocó en frascos transparentes de plástico de $1 \mathrm{~L}$ de capacidad, fijándose con formalina al $4 \%$ en una solución saturada de borato de sodio para su conservación final. De forma simultánea, en cada sitio de muestreo se obtuvieron los datos de temperatura y salinidad superficial por medio de un termómetro de inmersión graduado $\left(0,1^{\circ} \mathrm{C}\right.$ de precisión) y un refractómetro de campo (ATAGO s/mill-E, 1 psu de precisión), respectivamente.

El total de larvas de peces fue separado de las muestras e identificado hasta el nivel de especie cuando fue posible. Las principales fuentes bibliográficas utilizadas para la identificación fueron los trabajos de Sumida et al. (1985), Moser et al. (1993), Moser et al. (1994), Moser (1996) y Leis \& Carsons-Ewart (2000). Una vez identificado el material, se contabilizaron las larvas de cada especie y se normalizaron a un volumen de $1000 \mathrm{~m}^{3}$, mediante la siguiente fórmula: $\mathrm{N}=\mathrm{n} * 1000 / \mathrm{v}$. Donde $\mathrm{N}=$ abundancia normalizada, $\mathrm{n}=$ número de organismos y v = volumen filtrado del agua. Se calculó el índice de diversidad y equidad de Shannon H' (Magurran 1988) para cada uno de los sitios de muestreo y las estaciones del año, mediante la fórmula $\mathrm{H}^{\prime}=-\mathrm{S} \mathrm{Pi} \ln \mathrm{Pi}$, donde $\mathrm{Pi}=$ es la abundancia por especie proporcional del total encontrado. Asimismo fue aplicado el análisis de cluster entre las variables: especies-sitios de muestreo y especies-estaciones del año, con la finalidad de conocer el grado de afinidad o similitud entre éstas (Ludwing \& Reynolds 1988).

\section{Resultados}

\section{Características físicas}

Durante otoño e invierno se registraron los valores de temperatura más bajos $\left(25^{\circ} \mathrm{C}\right)$, en tanto que en el verano se registraron los valores más altos $\left(31,6^{\circ} \mathrm{C}\right)$; por otro lado, se registró una salinidad baja durante el verano (11 psu) en tanto que en el resto de las estaciones se registraron valores de 30 y hasta 34,8 psu (Fig. 2a). Respecto a las variaciones por sitio de muestreo, la temperatura fue menos variable que la salinidad. Los valores más bajos de temperatura se registraron en el sitio $1\left(27,4^{\circ} \mathrm{C}\right)$ y los más altos en el sitio $5\left(28,9^{\circ} \mathrm{C}\right)$. La salinidad mostró una variación muy pronunciada ya que se registraron valores desde 23,5 psu para la estación 1 y de 29 psu en la estación 5 (Fig. 2b).

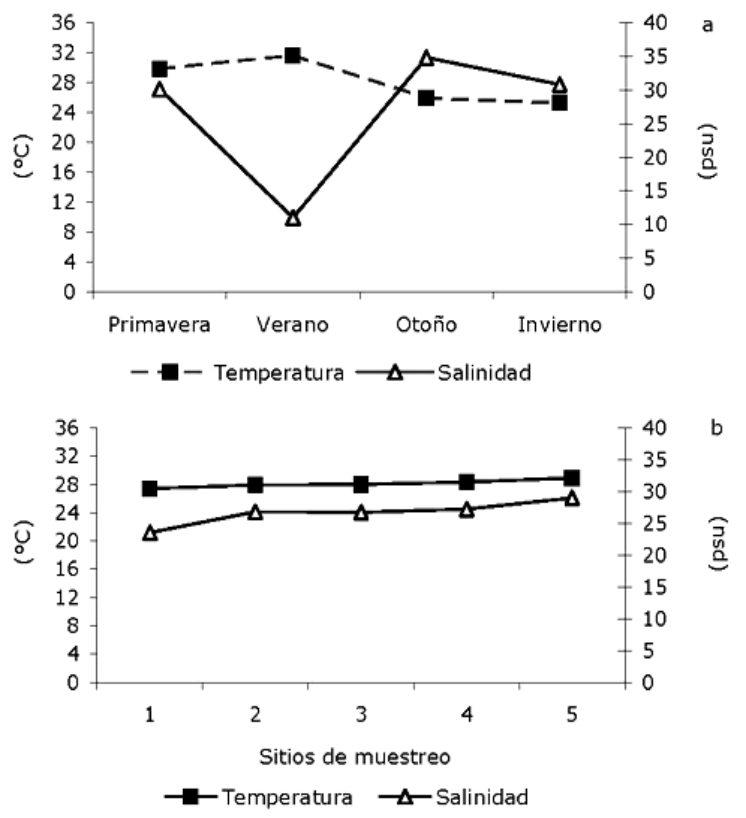

Figura 2

Variación promedio de la temperatura $\left({ }^{\circ} \mathrm{C}\right)$ y salinidad (psu) superficiales: a) por estación del año y b) por sitio de muestreo, en la laguna El Quelele, Nayarit, México (primavera-invierno 2001)

Mean variation of surface temperature $\left({ }^{\circ} \mathrm{C}\right.$ ) and salinity (psu):

a) by season and b) by sampling sites, in the laguna $\mathrm{El}$

Quelele, Nayarit, Mexico (spring-winter 2001)

\section{Larvas de peces}

Fueron recolectadas 224,4 larvas de peces, representadas por seis familias (Eliotridae, Carangidae, Gerreidae, Gobiidae, Engraulidae y Hemiramphidae), ocho géneros y seis especies, que por orden de importancia Engraulis mordax (Girard, 1856) fue la especie más abundante (68 org. $1000 \mathrm{~m}^{-3}$ ), seguida de Dormitator latifroms (Richardson, 1845) (37 org. 1000 $\mathrm{m}^{-3}$ ) y Trachinotus rhodopus (Gill, 1863) (18 org. 1000 $\mathrm{m}^{-3}$ ), en tanto el resto de las especies presentó de 1 a 5 org. $1000 \mathrm{~m}^{-3}$ (Fig. 3).

En cuanto a la distribución temporal, las mayores abundancias se registraron durante el otoño (81 org. $1000 \mathrm{~m}^{-3}$ ) e invierno (43 org. $1000 \mathrm{~m}^{-3}$ ) principalmente, y una menor concentración fue registrada en primavera (21 org. $1000 \mathrm{~m}^{-3}$ ), en tanto que en el verano no se obtuvieron larvas (Fig. 4). 


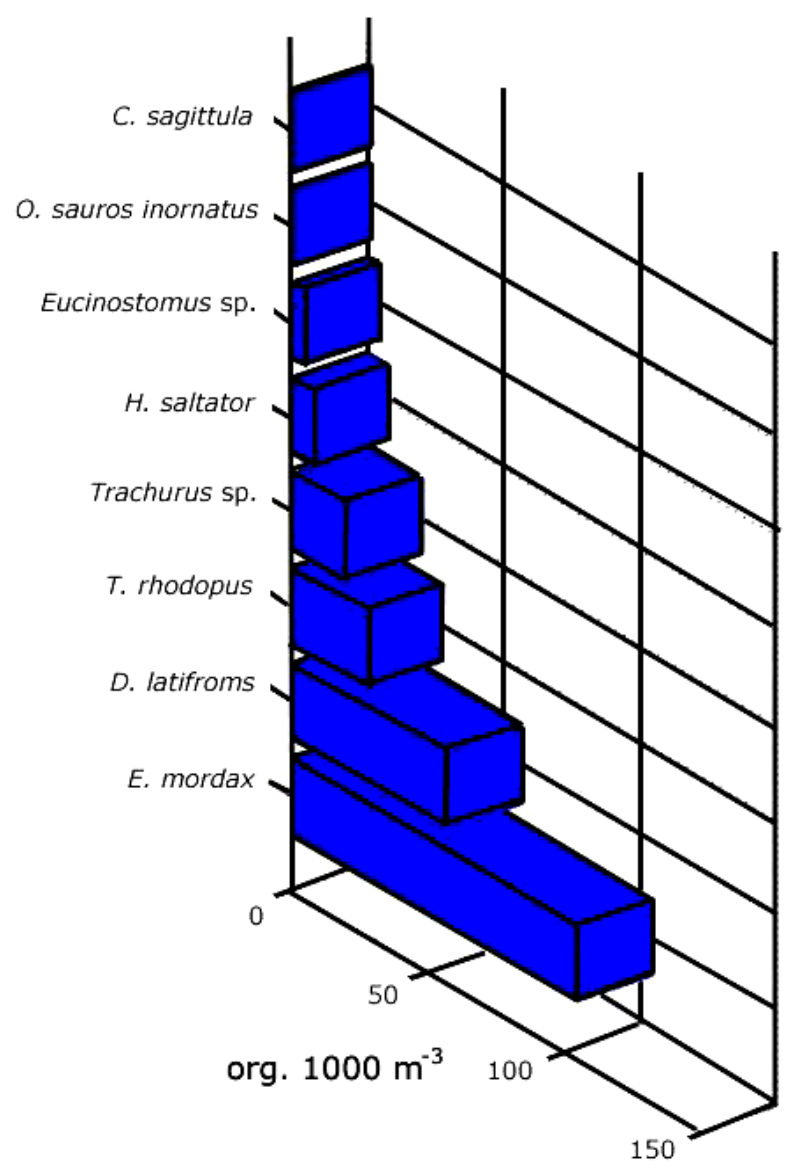

Figura 3

Abundancia (org. $1000 \mathrm{~m}^{-3}$ ) por especie (Engraulis mordax, Dormitator latifroms, Trachinotus rhodopus, Trachurus sp., Hemiramphus saltador, Eucinostomus sp., Oligoplites saurus inornatus, Ctenogobius sagittula) en la laguna El Quelele Nayarit, México (primavera-invierno 2001)

Abundance (org. $1000 \mathrm{~m}^{-3}$ ) by species (Engraulis mordax, Dormitator latifroms, Trachinotus rhodopus, Trachurus sp., Hemiramphus saltador, Eucinostomus sp., Oligoplites saurus inornatus, Ctenogobius sagittula) in the laguna El Quelele, Nayarit, Mexico (spring-winter 2001)

En lo que respecta a la variación de la abundancia del ictioplancton por sitios de muestreo, se observó que los sitios con mayores abundancias fueron el sitio 4 (55 org. $1000 \mathrm{~m}^{-3}$ ) seguido del sitio $2\left(31\right.$ org. $\left.1000 \mathrm{~m}^{-3}\right) \mathrm{y}$ posteriormente el 3 ( 23 org. $1000 \mathrm{~m}^{-3}$ ), en tanto que en los sitios 1 y 5 se registró una menor abundancia larval (13 y 20 org. $1000 \mathrm{~m}^{-3}$, respectivamente) (Fig. 5).

Referente a la variación espacial, se observó que $E$. mordax fue la especie con una mayor distribución en los sitios 3 y 5, seguida de $D$. latifroms con una distribución importante en los sitios 2 y 3; en cambio, Trachinotus rhodopus estuvo presente en el sitio 2, y Trachurus sp. fue registrada en el sitio 4; el resto de las especies presentó una distribución más limitada (Fig. 6).

En cuanto a la relación de la abundancia larval con la temperatura y salinidad, se observó que durante la primera mitad del año (época de estiaje) se encontraron mínimas concentraciones de larvas en primavera $(<10$ org. $1000 \mathrm{~m}^{-3}$ ) con registros bajos de salinidad (11 psu) y temperaturas moderadas $\left(30^{\circ} \mathrm{C}\right)$, en tanto que las mayores abundancias ( $>120$ org. $1000 \mathrm{~m}^{-3}$ ) fueron registradas en la segunda mitad del año (otoño principalmente), cuando los valores tanto de salinidad (35 psu) y temperatura $\left(26^{\circ} \mathrm{C}\right)$ eran altos; otra concentración importante fue observada durante el invierno, con abundancias mayores a 60 org. $1000 \mathrm{~m}^{-3}$, con temperaturas inferiores a $25^{\circ} \mathrm{C}$ y salinidades de 30 psu (Fig. 7).

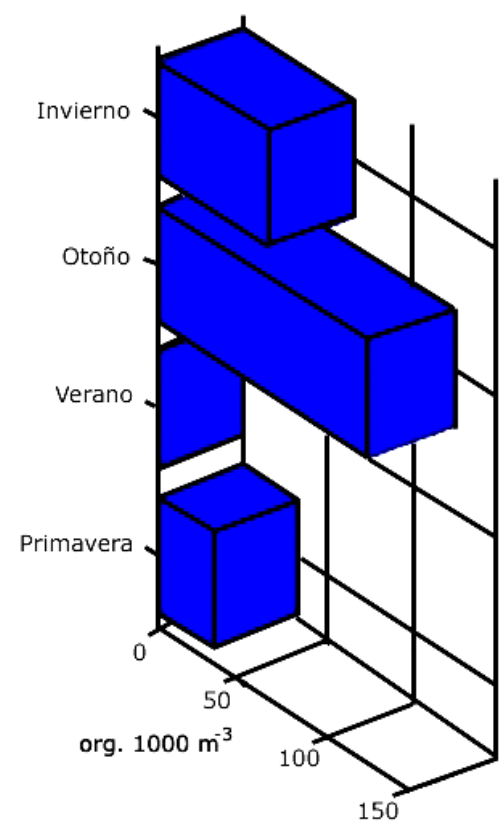

Figura 4

Variación temporal de la abundancia (org. $1000 \mathrm{~m}^{-3}$ ) del ictioplancton en la laguna El Quelele, Nayarit, México (primavera-invierno 2001)

Seasonal abundance variation (org. $1000 \mathrm{~m}^{-3}$ ) of ichthyoplankton in the laguna El Quelele, Nayarit, Mexico (spring-winter 2001) 


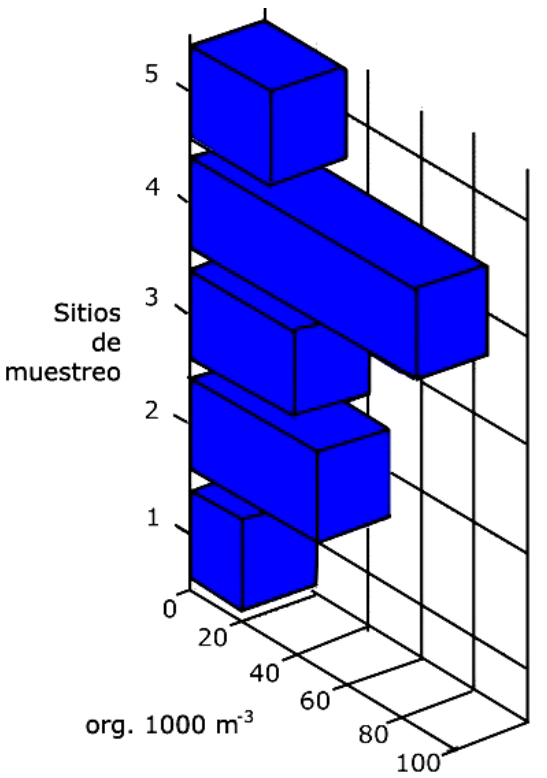

Figura 5

Variación de la abundancia (org. $1000 \mathrm{~m}^{-3}$ ) del ictioplancton por sitio de muestreo en la Laguna del Quelele, Nayarit, México (primavera-invierno 2001)

Abundance variation (org. $1000 \mathrm{~m}^{-3}$ ) of ichthyoplankton by sampling sites in the laguna El Quelele, Nayarit, Mexico

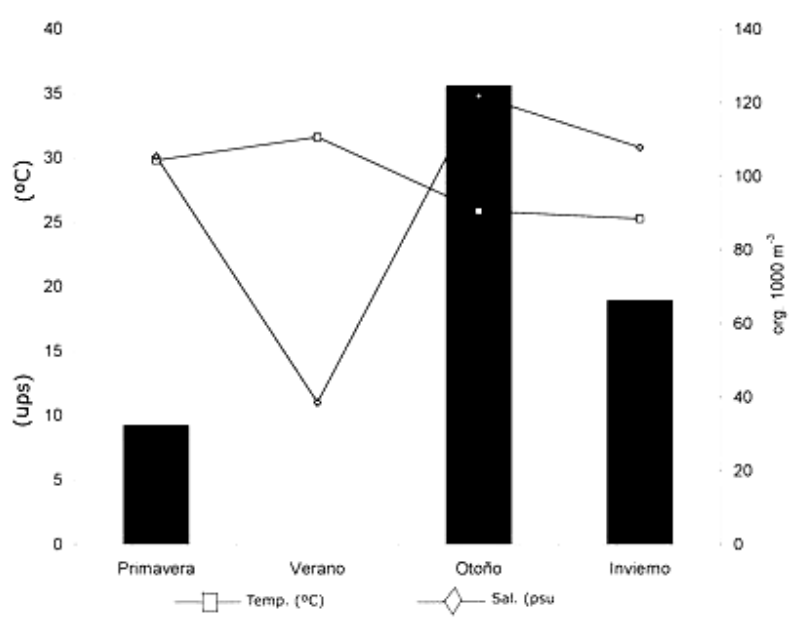

Figura 7

Variación promedio de la temperatura superficial $\left({ }^{\circ} \mathrm{C}\right)$, salinidad (psu) y abundancia (org. $1000 \mathrm{~m}^{-3}$ ) en la laguna El Quelele Nayarit, México (primavera-invierno 2001)

Mean variation of surface temperature $\left({ }^{\circ} \mathrm{C}\right)$, salinity (psu) and abundance (org. $1000 \mathrm{~m}^{-3}$ ) in the laguna El Quelele, Nayarit, Mexico (spring-winter 2001)

(spring-winter 2001)

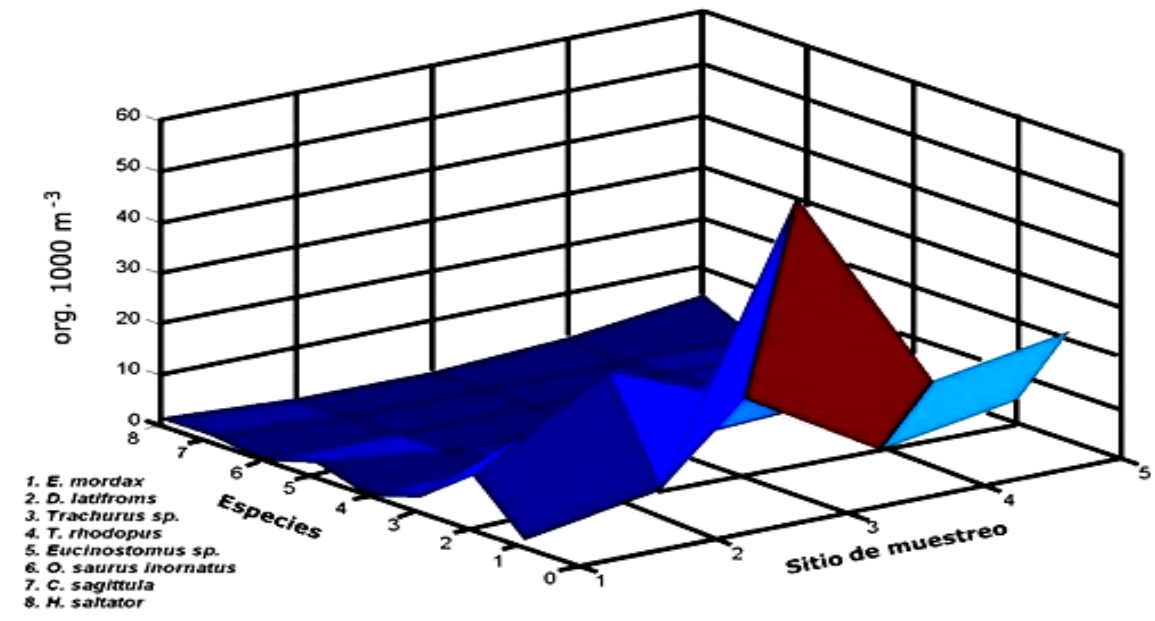

Figura 6

Variación espacial de la distribución y abundancia (org. $1000 \mathrm{~m}^{-3}$ ) por especie (Engraulis mordax, Dormitator latifroms, Trachurus sp, Trachinotus rhodopus, Eucinostomus sp., Oligoplites saurus inornatus, Ctenogobius sagittula y Hemiramphus saltator), por sitio de muestreo y estación del año, en la laguna El Quelele, Nayarit, México (primavera-invierno 2001)

Spatial variation of distribution and abundance (org. $1000 \mathrm{~m}^{-3}$ ) by species (Engraulis mordax, Dormitator latifroms, Trachurus sp., Trachinotus rhodopus, Eucinostomus sp., Oligoplites saurus inornatus, Ctenogobius sagittula and Hemiramphus saltator), by sampling site and season in the laguna El Quelele, Nayarit, Mexico (spring-winter 2001) 
Respecto del análisis de diversidad por sitio de muestreo (espacial), en la estación 1 se registró el valor máximo $(1,47)$, en tanto que el valor mínimo $(0,82)$ se presentó en el sitio 5. En cuanto a la diversidad por estación del año (temporal), en otoño se observó el valor máximo $(1,21)$, en tanto que en invierno se reportaron los valores mínimos $(1,10)$ (Tabla 1$)$.

\section{Tabla 1}

Índice de diversidad de Shannon por sitio de muestreo y estación del año en la laguna El Quelele, Nayarit, México (primavera-invierno 2001)

Shannon diversity index by sampling site and season in the laguna El Quelele (spring-winter 2001)

\begin{tabular}{cccccc}
\hline $\begin{array}{c}\text { Sitio de muestreo } \\
\text { (espacial) }\end{array}$ & 1 & 2 & 3 & 4 & 5 \\
\cline { 2 - 6 } Índice & 1,47 & 1,06 & 0,84 & 1,26 & 0,82 \\
& & & & & \\
$\begin{array}{c}\text { Estación del año } \\
\text { (temporal) } \\
\text { Índice }\end{array}$ & Primavera Verano & Otoño & Invierno & \\
\cline { 2 - 6 } & 1,15 & $*$ & 1,21 & 1,1 & \\
\hline
\end{tabular}

El análisis de cluster aplicado a las variables especies-sitios de muestreo mostró una mayor afinidad entre los sitios 2 y 3 (1,05) mientras que en los sitios 1 y 5 , la afinidad fue menor $(1,42)$; el sitio 4 es el que menos afinidad presentó con el resto de los sitios $(1,89)$ (Fig. 8a). Referente al análisis entre las variables especies-estación del año, se encontraron dos grupos con afinidades entre las estaciones frías (otoño-invierno) con un valor de 0,83 y las estaciones cálidas (primaveraverano) con un valor de 1,79 (Fig. 8b).

\section{Discusión}

Las variaciones en temperatura, salinidad y abundancia del ictioplancton indican que cuando se presentan las temperaturas bajas $\left(25^{\circ} \mathrm{C}\right)$ en otoño e invierno se registra una mayor salinidad (30-34,8 psu) y una mayor abundancia de larvas (43-81 org. $1000 \mathrm{~m}^{-3}$ ), la que se asocia a la época fría. En tanto que, al aumentar la temperatura $\left(31,6^{\circ} \mathrm{C}\right)$ y disminuir la salinidad (11 psu) se presenta un decremento de la abundancia y diversidad larval, asociándose a la época cálida con abundantes lluvias. Ésto sugiere que pudiese existir una relación estrecha e inversamente proporcional entre las variaciones de temperatura y salinidad con la abundancia larval, es decir, a menor temperatura $y$
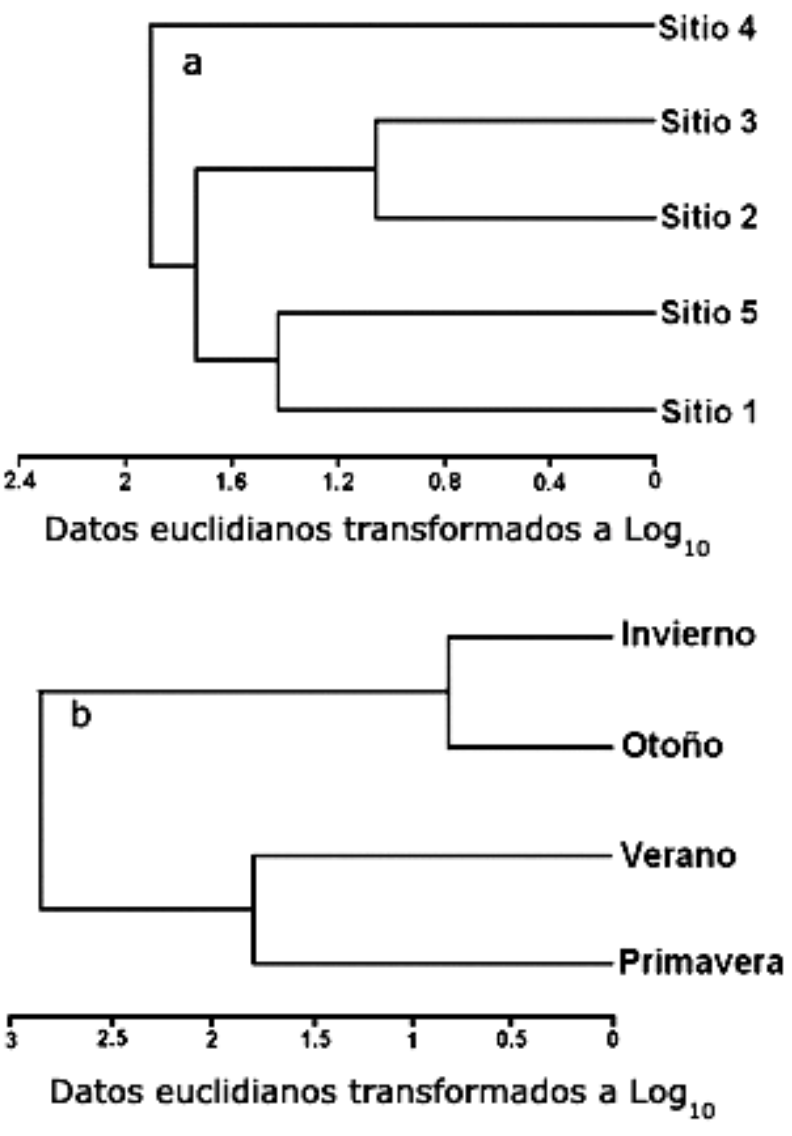

Figura 8

Análisis de cluster. a) especies-sitios de muestreo; b) especies-estación del año

Cluster analysis. a) species-sampling sites; b) species-season

mayor salinidad se presenta un aumento en la abundancia y diversidad, en tanto que a mayor temperatura y menor salinidad disminuye considerablemente la abundancia y diversidad larval. Margalef (1969) y Livingston (1984) señalan que el zooplancton, en general, en las lagunas costeras presenta una baja diversidad, situación que se ha asociado con la alta variabilidad del sistema en términos de salinidad y/o temperatura. Por su parte, NavarroRodríguez et al. (2004) indican que en el sistema estuarino El Salado (sistema que se localiza a una distancia no mayor de $15 \mathrm{~km}$ del sistema El Quelele), se presenta un patrón de distribución en espacio y tiempo 
totalmente diferente al del sistema El Quelele; los autores señalan que las variaciones de temperatura, salinidad y abundancia de larvas de peces están en estrecha relación con el patrón estacional, influyendo a su vez en el ciclo de reproducción de las especies, ya que se presentaron las mayores abundancias $(12,000$ org. $1000 \mathrm{~m}^{-3}$ ) con el incremento de la temperatura y una disminución de la salinidad $\left(20-30^{\circ} \mathrm{C}\right.$ y 6,5 psu); tambien indican que las variaciones de la abundancia espacio-temporal estuvieron influenciadas tanto por las condiciones de marea como por la época del año, debido a que las mayores concentraciones de larvas fueron asociadas a la época de lluvias durante el verano, en tanto que, en primavera se presentaron los registros más bajos durante todo el periodo de estudio asociado con la época de sequía. Por otro lado, la diversidad fue mínima ya que se obtuvieron registros del 97,8\% para Dormitator latifroms, en tanto que solo el 2,2\% correspondió a otras especies; es relevante señalar que el período de estudio fue llevado a la par en ambos sistemas.

Es posible que estas diferencias se deban principalmente, a las características naturales propias de cada sistema y las continuas modificaciones a las que están sujetos, y así como a los hábitos y épocas reproductivas de las distintas especies. Las especies marinas fueron las que dominaron en el sistema El Quelele (menos impactado) en tanto que en el estero El Salado (altamente impactado), la especie dominante es principalmente de hábitos estuarinos, a pesar de que ambos cuerpos de agua se localizan en la misma zona climática (semicálida subhúmeda y se encuentran bajo el mismo régimen de mareas.

El hecho de encontrar especies de ambientes marinos en El Quelele puede indicar que sus abundancias estuvieron asociadas al transporte por las corrientes, patrones de circulación locales y a los efectos de mareas, utilizando estos sistemas de alta productividad primaria como áreas de reproducción, crianza, alimentación y protección contra sus depredadores. Esto mismo ha sido indicado por Álvarez-Cadena et al. (1984), Day \& Yáñez Arancibia (1985) y Contreras (1993). En lo que respecta a los patrones de circulación y corrientes, Torres-Orozco (1994) señala que los sistemas estuarinos son muy complejos y están fuertemente influenciados por las mareas y las fluctuaciones en el aporte de agua dulce de los sistemas hídricos adyacentes, además de los vientos y la geomorfología, ocasionando de alguna manera la sustitución de unas especies por otras, incluso dentro de una misma familia, hasta terminar con el reemplazo de familias completas, o bien, incrementando la distribución de algunas otras especies.

Por otro lado, se observó que las variaciones espacio-temporales de la abundancia larval de estas especies marinas pudiesen estar influenciadas o asociadas a dos factores más: la lejanía y cercanía de los sitios de muestreo con la entrada permanente de agua de mar a este sistema estuarino. Esto es, que la abundancia larval se vio disminuida principalmente en el sitio 1 (lejana), en tanto que en el sitio 4 (cercana), el aumento de la abundancia fue notorio, lo que significa que el $68 \%$ de la abundancia total se presentó en las estaciones abiertas o cercanas al mar en tanto que en las más alejadas solo se reportó el 32\%. Rodríguez (1975) señala que en los sistemas costeros existe generalmente, un frente salino cuya ubicación depende de la intensidad de los aportes de agua dulce y de la amplitud de la marea, formándose en consecuencia una zonificación. Dicha zonificación es caracterizada faunísticamente: la zona oligohalina es una zona con mayor influencia de agua dulce (5 psu) presentándose una biomasa baja; la zona de mezcla donde las corrientes de marea determinan la penetración moderada de los zoopláncteres y cuya salinidad es mayor (7-15 psu), la zona crítica que presenta salinidades de 15-20 psu, con una biomasa elevada y donde la fauna dominante es de tipo nerítico-marino y finalmente, la zona nerítica cuya salinidad es marina por arriba del 30 psu, donde el factor dominante son las corrientes y contracorrientes marinas, presenta especies plenamente marinas. A pesar de esta caracterización que señala el autor, en este trabajo encontramos que desde el sitio 1, que recibe más aporte de agua dulce que salada, hasta el sitio 5 que prácticamente estuvo localizado en la boca, recibiendo un mayor aporte de agua salada, se registraron especies totalmente marinas; sin embargo, en lo que respecta a las abundancias, Rodríguez (1975) señala que en la zona (crítica) con salinidades 15-20 psu, se presenta una biomasa elevada, situación que nosotros también observamos ya que se presentó el 68\% del total de la abundancia en los sitios donde ocurre una marcada mezcla entre agua salada y dulce. Consideramos que, en este caso en particular, y de acuerdo a los porcentajes de abundancia, los sitios abiertos de la laguna El Quelele pudiesen ser la zona principal de desove, al menos, de E. mordax, D. latifroms y Trachurus sp. Otra situación asociada a dichas variaciones estaría dada por el tipo de distribución que presenta el ictioplancton en general, ya que ésta se da a manera de parches y no de una forma dispersa, por lo que, en cierta medida, influye en la 
ampliación o limitación de su distribución, como fue evidente en los sitios 1 (menor distribución) y 4 (mayor distribución) durante todo el período de muestreo. Suárez- Morales (1994) señala que la distribución, en general, del zooplancton no es uniforme en un sistema costero sino que existen elementos para afirmar que la distribución, se establece en parches, de modo que en ciertos espacios donde las condiciones son adecuadas, el zooplancton tiende a concentrarse.

Asimismo, el hecho de no haber registrado larvas de peces durante el verano, pudiese estar asociado principalmente, a que, en esta época, se presentan los valores más bajos de salinidad (11 psu), situación que es atribuida a las lluvias ocasionadas por la presencia de ciclones y al aporte de agua dulce proveniente de las escorrentías aledañas al área de estudio.

Por otro lado, fueron observadas claramente las afinidades entre los sitios de muestreo 2-3 y 1-5 los cuales compartieron generalmente las mismas especies; sin embargo, el sitio 4 fue totalmente diferente del resto de los sitios. Esto sugiere que el sitio 4, dada su ubicación (entre dos puentes vehiculares), pudiera estar bajo efectos de impacto antropogénico más directo que el resto de los sitios, a pesar de que fue el sitio que registró las abundancias más altas pero únicamente de dos especies (E. mordax con 38 org. $1000 \mathrm{~m}^{-3} \mathrm{y}$ Trachurus sp. con 10 org. $1000 \mathrm{~m}^{-3}$ ), en tanto que los sitios restantes presentaron abundancias menores pero con una mayor diversidad. De igual manera, fue observado entre las estaciones del año afinidades entre las estaciones cálidas (primavera-verano) y las frías (otoño-invierno), lo que sugiere la clara presencia de dos épocas reproductivas con picos máximos durante la temporada fría, al menos, para E. mordax (42 org. 1000 $\mathrm{m}^{-3}$ ) y D. latifroms, (26 org. $1000 \mathrm{~m}^{-3}$ ). En lo que respecta a $D$. latifroms, Navarro-Rodríguez et al. (2004) indican, de acuerdo a los resultados obtenidos en el sistema El Salado, que el período reproductivo de esta especie podría estar comprendido entre el verano y el otoño, de acuerdo a los picos de abundancias que presentó la especie; en tanto que, para E. mordax, es conocido que desova entre diciembre y junio y que, sin embargo, es una especie que es capturada generalmente muy al norte del Pacífico mexicano incluyendo el Golfo de California (Whitehead et al. 1995), por lo que no es común encontrarla en estas latitudes; sin embargo, no significa que no pueda presentarse en estas áreas, ya que la corriente costera de Costa Rica, durante junio y julio, sigue la costa de Centroamérica y México (donde se le conoce como Corriente mexicana), hasta alcanzar la zona de transición del Pacífico Norte, frente al extremo de la península de Baja California y, que conjuntamente, giran para realimentar el sistema ecuatorial (Badan 1997), lo que favorece de alguna manera, la distribución de esta especie hasta esta área de estudio, ya que las fechas de desove y la de la presencia de este evento coinciden en cierto tiempo, lo que pudiera explicar los picos de abundancias de E. mordax en el área de estudio en la época cálida, pero principalmente, en la época fría.

Es un hecho que las características propias de los sistemas estuarinos favorecen la crianza de las larvas de peces, dado que existe una baja energía y una elevada productividad determinando que, en la mayoría de los casos, estos sistemas resulten verdaderos viveros para numerosas especies de peces; sin embargo, no hay que pasar por alto que si bien estas larvas se desarrollan en estos sistemas, no todas la especies permanecen en ellos durante su etapa adulta, retornando a las aguas marinas, para después volver a los sistemas estuarinos a desovar.

\section{Agradecimientos}

Los autores agradecemos los comentarios y sugerencias de los revisores para mejorar este manuscrito.

\section{Literatura citada}

Álvarez-Cadena JN, MA Aquino, F Alonso, JG Millán \& T Torres. 1984. Composición y abundancia de las larvas de peces en el sistema lagunar Huizache-Caimanero. Parte I Agua dulce 1978. Anales del Instituto de Ciencias del Mar y Limnología, Universidad Nacional Autónoma de México, México D.F. 11(1):163-180.

Badan A. 1997. Corriente Costera de Costa Rica en el Pacífico Mexicano. En: Lavín MF (ed), Contribuciones a la Oceanografía Física en México, Monografía 3: 99112, Unión Geofísica Mexicana.

Contreras EF. 1993. Ecosistemas Costeros Mexicanos $1^{\text {a }}$ ed. Comisión Nacional para el Conocimiento y Uso de la Biodiversidad. Universidad Autónoma Metropolitana, Unidad Iztapalapa. 415 pp.

Cupul-Magaña FG. 1999. La laguna El Quelele, Nayarit, México, como hábitat de aves acuáticas. Revista Ciencia y Mar 3(8): 21-28.

Cupul-Magaña FG. 2000. Notas sobre la avifauna acuática de las islas y los humedales costeros de Bahía de Banderas, Jalisco-Nayarit, México. Revista Mexicoa 2(1): 85-92.

Day JW \& A. Yañez-Arancibia. 1985. Coastal lagoons and estuaries as an environment nekton, En: Yañez-Arancibia 
A (ed). Fish Community Ecology in Estuaries and Coastal Lagoons: Towards Ecosystem Integration, 3: 17-34. Universidad Nacional Autónoma de México, México, D.F.

Flores Verdugo F.1989. Algunos aspectos sobre la ecología, uso e importancia de los ecosistemas de manglar. En: de la Rosa-Vélez J \& F González-Farias (eds). Temas de Oceanografía Biológica en México. Pp. 21-56. Universidad Autónoma de Baja California, México.

Funes Rodríguez R, MA Fernández Álamo \& R González Armas. 1998. Larvas de peces recolectadas durante dos eventos El Niño en la costa occidental de Baja California Sur, México, 1958-1959 y 1983-1984. Oceánides 13(1): $67-75$.

García E. 1973. Modificaciones del Sistema de Clasificación Climática de Köppen (para adaptarlas a las condiciones de la República Mexicana). Instituto de Geografía, Universidad Nacional Autónoma de México, 2ª ed, 246 pp.

Gobierno del Estado de Jalisco. 1999. Ordenamiento ecológico de la región Costa de Jalisco. Periódico Oficial del Estado de Jalisco. Tomo CCXXXI. 83 pp.

Leis JM \& BM Carson-Ewart. 2000. The larvae of IndoPacific coastal fishes: An identification guide to marine fish larvae. Fauna Malesiana Foundation and National Museum of Natural History. Pp. 1-883.

Livingston RJ. 1984. The ecology of the Apalachicola Bay system: an estuarine profile. U.S. Fish Wildlife Service Off. Biological Services (Technical Reports) FWS/OBS: 82-105.

Ludwing JA \& JF Reynolds. 1988. Statistical Ecology: A Primer on methods and computing. 337 pp. John Wiley \& Sons, Nueva York.

Magurran AE. 1988. Ecological Diversity and its Measurement. 179 pp. Princenton University Press. New Jersey.

Margalef R. 1969. Comunidades planctónicas en lagunas litorales. Pp. 545-562, Memorias del Simposio Internacional de Lagunas Costeras, UNAM-UNESCO (1967). México Distrito Federal.

Moser HG \& PE Smith. 1993. Larval fish assemblages of the California Current region and their horizontal and vertical distributions across a front. En: Advances in the early life history of fishes, part 1-2. Bulletin of Marine Science 53(2): 645-691.

Moser HG, RL Charter, PE Smith, DA Ambrose, SR Charter, CA Meyer, EM Sandknop \& W Watson. 1994. Distributional atlas of fish larvae and eggs in the California Current region: taxa with less than 1000 total larvae, 1951 through 1984. California Cooperative Oceanic Fisheries Investigations Atlas 32, 181 pp.

Moser HG. 1996. The early stages of fishes in the California Current region. California Cooperative Oceanic Fisheries Investigation Atlas 33, 1505 pp.

Navarro-Rodríguez MC, R Flores-Vargas, LF González Guevara \& ME González Ruelas. 2004. Distribution and abundance of Dormitator latifroms (Richardson) larvae (Pisces: Eliotridae) in the natural protected area "estero El Salado” in Jalisco, Mexico. Revista de Biología Marina y Oceanografía 39(1): 31-36.

Rodríguez G. 1975. Some aspects of the ecology of tropical estuaries. En: Golley F \& E Medina (eds). Ecological Studies. Tropical Ecological Systems 2: 313-333. Springer-Veriag, Nueva York.

Suárez Morales E. 1994. Comunidades zooplanctónicas de las lagunas costeras. En: De la Lanza Espino G \& C Cáceres Martínez (eds). Lagunas Costeras y el Litoral Mexicano. $1^{\text {a }}$ ed, pp. 246-268. Universidad Autónoma de Baja California Sur, Universidad Nacional Autónoma de México.

Sumida BY, HG Moser \& EH Ahlstrom. 1985. Descriptions of larvae of California yellowtail, Seriola lalandi and the three other carangids from the Eastern Tropical Pacific: Chloroscombrus orqueta, Caranx caballus and Caranx sexfasciatus. California Cooperative, Oceanic Fisheries Investigation Reports 26:139-159.

Torres-Orozco R. 1994. Los Peces. En: De la Lanza Espino G \& C Cáceres Martínez (eds). Lagunas Costeras y el Litoral Mexicano. $1^{\mathrm{a}}$ ed, pp. 269-304. Universidad Autónoma de Baja California Sur, Universidad Nacional Autónoma de México.

Whitehead, PJ \& R Rodríguez-Sánchez. 1995. Engraulidae. En: Fischer W, F Krupp, W Schneider, C Sommer, KE Carpenter \& VH Niem (eds). Guía FAO para la identificación de especies para los fines de la pesca. Pacífico Centro-Oriental. Vols. II y III Partes 1 y 2: 10671068. FAO, Roma. 